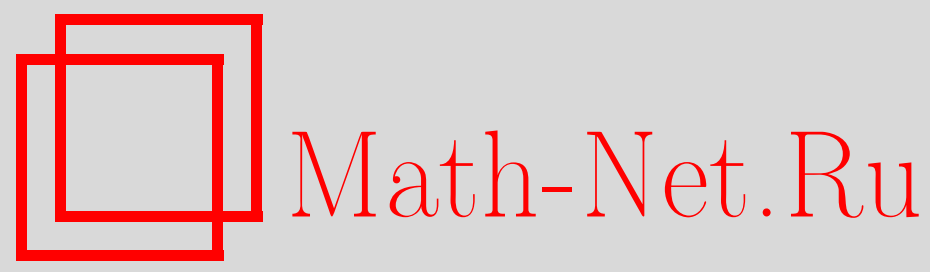

Г. Г. Бильченко, И. Р. Каюмов, С. Р. Насыров, М. Ф. Садыков, Разветвленные накрытия неориентируемых поверхностей ориентируемыми с заданной проекцией края, Матем. заметки, 1998, том 63, выпуск 2, 292-294

DOI: https://doi.org/10.4213/mzm1276

Использование Общероссийского математического портала Math-Net.Ru подразумевает, что вы прочитали и согласны с пользовательским соглашением http://www . mathnet.ru/rus/agreement

Параметры загрузки:

IP : 44.207 .124 .84

26 апреля 2023 г., 10:07:34

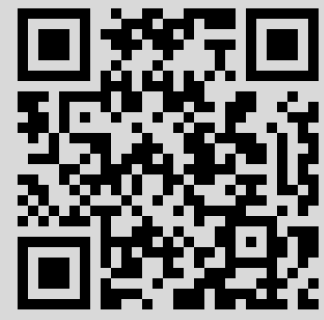




\section{РАЗВЕТВЛЕННЫЕ НАКРЫТИЯ НЕОРИЕНТИРУЕМЫХ ПОВЕРХНОСТЕЙ ОРИЕНТИРУЕМЫМИ С ЗАДАННОЙ ПРОЕКЦИЕЙ КРАЯ}

\section{Г. Г. Бильченко, И.Р. Каюмов, С.Р. Насыров, М. Ф. Садыков}

Пусть $N$-компактная поверхность (без края), $\bar{M}=M \cup \partial M-$ компактная поверхность с краем, $p: \bar{M} \rightarrow N$ - непрерывное внутреннее в смысле Стоилова (см., например, [1, гл. V]) отображение. Пусть $\alpha_{1}, \ldots, \alpha_{\nu}$ - кривые, которые обходят компоненты края $\partial \mathrm{M} \mathrm{в} \mathrm{положительном} \mathrm{направлении:}$ $\beta_{1}=p\left(\alpha_{1}\right), \ldots, \beta_{\nu}=p\left(\alpha_{\nu}\right)$. Будем говорить, что пара $\sigma=(\bar{M}, p)$ есть поверхность над $N$, ограниченная кривыми $\beta_{1}, \ldots, \beta_{\nu}$. Представляет интерес нахождение условий, которым должны удовлетворять кривые $\beta_{1}, \ldots, \beta_{\nu}$, для того чтобы существовала ориентируемая поверхность $\sigma=(\bar{M}, p)$ над $N$, ограниченная этими кривыми, в случае, когда $N$ неориентируема.

Эта задача является обобщением классических задач, поставленных Пикаром, Левнером и Хопфом и изучавшихся для случая, когда $N$ ориентируема, в работах Титуса, М. Маркса, Френсиса, Эзелля и др. (см. подробнее [2], [3]). В неориентируемом случае Эзелль [4] исследовал эту задачу комбинаторными методами. В данной работе с использованием результатов из [3] для случая одной граничной кривой получено решение, формулирующееся в алгебраических терминах.

Пусть $\infty_{N}-$ фиксированная точка из $N$. Введем множество $\mathfrak{M}^{*}$ замкнутых кривых на компактной поверхности $N$.

ОПРЕДЕЛЕНИЕ 1 . Кривая $\beta \in \mathfrak{M}^{*}$, если выполнены следующие условия:

1) $\beta$ локально проста, т.е. для любого пути $z:[0,1] \rightarrow N$, представляющего $\beta$, и любого $t \in[0,1]$ 1-периодическое продолжение $\widetilde{z}: \mathbb{R} \rightarrow N$ пути $z$ инъективно в достаточно малой окрестности точки $t$;

2 ) носитель $|\beta|$ кривой $\beta$ разбивает $N$ на конечное число частей;

3) $\beta$ не проходит через $\infty_{N}$;

4) если $\widetilde{z}\left(t_{1}\right)=\widetilde{z}\left(t_{2}\right)=\widetilde{z}\left(t_{3}\right)=z_{0}$, где $t_{1}<t_{2}<t_{3},\left|t_{1}-t_{3}\right| \leqslant 1$, то при достаточно малом $r>0$ кривые $\omega_{1}$ и $\omega_{2}$ с представлениями

$$
z_{i}(t)=\left\{\begin{array}{ll}
\widetilde{z}\left(r(t-1 / 2)+t_{i}\right), & 0 \leqslant t \leqslant 1 / 2, \\
\widetilde{z}\left(r(t-1 / 2)+t_{i+1}\right), & 1 / 2 \leqslant t \leqslant 1,
\end{array} \quad i=1,2\right.
$$

являются простыми и существуют простые кривые $\zeta_{1}$ и $\zeta_{2}$ такие, что для некоторой ориентации в окрестности точки $z_{0}$ кривая $\zeta_{1}$ подходит “слева" к обеим кривым $\omega_{1}, \omega_{2}$ в точке $z_{0}$, а $\zeta_{2}$ - "справа".

Пусть $\sigma=(\bar{M}, p)$ есть поверхность над $N$, ограниченная кривой $\beta$. Как и в [5], нетрудно показать, что для любой точки $P \in N \backslash|\beta|$ число прообразов $P$ при отображении $p$ конечно. В достаточно малой окрестности любой точки $Q \in p^{-1}(P)$ внутреннее отображение $p$ по теореме Стоилова $[1$, с. 152$]$ топологически эквивалентно степенному отображению $z \mapsto z^{n}$ в окрестности начала координат комплексной плоскости. Число $\operatorname{ord}(Q, \sigma)=n-1$ назовем кратностью ветвления поверхности $\sigma$ в точке $Q$. По определению число листов поверхности $\sigma$ над точкой $P$ (с учетом кратности ветвления) есть

$$
n_{\sigma}(P)=\sum_{Q \in p^{-1}(P)}(\operatorname{ord}(Q, \sigma)+1)
$$

Работа выполнена при частичной поддержке Российского фонда фундаментальных исследований, грант № 96-01-00110. 
Рассмотрим двулистное ориентируемое накрытие $\widetilde{N}$ поверхности $N$ с накрывающим отображением $\psi: \widetilde{N} \rightarrow N$. Пусть $a^{\prime} \in M, a \in N, \widetilde{a} \in \widetilde{N}$ - точки, для которых $\psi(\widetilde{a})=a=p\left(a^{\prime}\right)$. Тогда нетрудно видеть, что существует накрьвающее отображение $\varphi: \bar{M} \rightarrow \widetilde{N}$ такое, что диаграмма

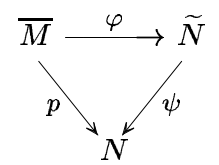

коммутативна и $\varphi\left(a^{\prime}\right)=\widetilde{a}$.

ОПРЕДЕЛЕНИЕ 2 . Замкнутую кривую $\beta$ в $N$ назовем кривой, сохраняющей ориентацию, если ее поднятие на $\widetilde{N}$ замкнуто. Кривую, не сохраняющую ориентацию, будем называть кривой, меняющей ориентацию.

В дальнейшем через $\pi_{1}(X, x)$ будем обозначать фундаментальную группу топологического пространства $X$ в точке $x$, через $[\omega]$ - гомотопический класс кривой $\omega$, а через $f_{\sharp}$ - гомоморфизм фундаментальных групп, индуцированный отображением $f$.

Пусть $\beta \in \mathfrak{M}^{*}$, тогда $\beta$ разбивает $N$ на $l+1$ область $G_{0}, \ldots, G_{l}$. В каждой из областей $G_{j}$ выберем по точке: $\infty_{N}=b_{0} \in G_{0}, \ldots, b_{l} \in G_{l}$. Пусть $a$ - начало кривой $\beta, A=N \backslash\left\{b_{0}, \ldots, b_{l}\right\}$, $\gamma_{0}, \ldots, \gamma_{l}$-простые петли в $A$ с началом в точке $a$, сохраняющие ориентацию, попарно не пересекающиеся (за исключением точки $a$ ), причем каждая из кривых $\gamma_{i}$ ограничивает в $N$ некоторую жорданову область $D_{i}$, которая содержит точку $b_{i}$ и не содержит точек $b_{j}$ при $j \neq i$.

Фиксируем некоторую ориентацию на $\widetilde{N}$. Пусть $\psi^{-1}(a)=\{\widetilde{a}, \widetilde{\widetilde{a}}\}$. Обозначим через $\gamma_{i}^{(1)}$ и $\gamma_{i}^{(2)}$ кривые, получающиеся поднятием кривой $\gamma_{i}$ на $\widetilde{N}$ из точек $\widetilde{a}$ и $\widetilde{\widetilde{a}}$ соответственно. Так как область $D_{i}$ односвязна, то $\psi^{-1}\left(D_{i}\right)=D_{i}^{(1)} \sqcup D_{i}^{(2)}$, причем область $D_{i}^{(1)}$ ограничена кривой $\gamma_{i}^{(1)}$, а $D_{i}^{(2)}-$ кривой $\gamma_{i}^{(2)}$. Пусть $b_{i}^{(1)} \in D_{i}^{(1)}, b_{i}^{(2)} \in D_{i}^{(2)}$-прообразы точки $b_{i} ; \widetilde{A}=\widetilde{N} \backslash\left\{b_{i}^{(1)}, b_{i}^{(2)} \mid i=0, \ldots, l\right\}$.

Выберем направление обхода кривых $\gamma_{0}, \ldots, \gamma_{l}$ так, чтобы кривая $\gamma_{i}^{(1)}$ обходила границу области $D_{i}^{(1)}$ в положительном направлении. Тогда очевидно, что кривая $\gamma_{i}^{(2)}$ обходит границу области $D_{i}^{(2)}$ в отрицательном направлении.

Пусть $[\pi, \pi]$ - коммутант группы $\pi_{1}(A, a)$, т.е. подгруппа в $\pi_{1}(A, a)$, порожденная всевозможными коммутаторами. (Напомним, что коммутатором элементов $x$ и $y$ назьвается элемент $[x, y]=$ $x y x^{-1} y^{-1}$.) Для любого $[\delta] \in[\pi, \pi]$ обозначим через $\Delta([\delta], \rho)$ множество наборов $d=\left\{\left[\delta_{i j}\right] \mid\right.$ $i=1,2, j=1, \ldots, \rho\}$ элементов из $\pi_{1}(A, a)$ таких, что $[\delta]$ представим в виде произведения $\rho$ коммутаторов

$$
[\delta]=\prod_{j=1}^{\rho}\left[\left[\delta_{1 j}\right],\left[\delta_{2 j}\right]\right] .
$$

Если $[\delta]=e-$ единичный элемент, то по определению полагаем $\Delta([\delta], 0)=\{e\}$. Теперь определим степень әлемента $[\delta]$ :

$$
\operatorname{deg}[\delta]=\min \{\rho \in \mathbb{Z} \mid \Delta([\delta], \rho) \neq \varnothing\} .
$$

Обозначим через $\pi^{+}(A, a)$ полугруппу в $\pi_{1}(A, a)$, порожденную элементами вида $[\xi]\left[\gamma_{j}\right][\xi]^{-1}$, $j=1, \ldots, l$, где $[\xi] \in \pi_{1}(A, a)$ и $\xi$ сохраняет ориентацию, а также элементами вида $[\eta]\left[\gamma_{j}\right]^{-1}[\eta]^{-1}$, $j=1, \ldots, l$, где $\eta$ меняет ориентацию. Пусть $\pi_{p}^{n}$ - множество элементов вида

$$
\prod_{i=1}^{n}\left[\xi_{i}\right]\left[\gamma_{0}\right]\left[\xi_{i}\right]^{-1},
$$

где $\left[\xi_{i}\right] \in \pi_{1}(A, a)$ и $\xi_{i}$ сохраняют ориентацию, $\pi_{r}^{n}$ - множество элементов вида

$$
\prod_{i=1}^{n}\left[\eta_{i}\right]\left[\gamma_{0}\right]^{-1}\left[\eta_{i}\right]^{-1}
$$


где $\left[\eta_{i}\right] \in \pi_{1}(A, a)$ и $\eta_{i}$ меняют ориентацию.

Обозначим через $\Sigma_{n}(\beta, \rho)$ класс ориентируемых поверхностей $\sigma$ над $N$ рода $\rho$, ограниченных кривой $\beta$, для которых число листов над бесконечно удаленной точкой (с учетом кратности ветвления) $n_{\sigma}\left(\infty_{N}\right)=n$, и положим по определению

$$
S_{n}\left(\beta, \rho_{0}\right)=\bigcup_{\rho \leqslant \rho_{0}} \Sigma_{n}(\beta, \rho) .
$$

Теорема. Пусть $\beta \in \mathfrak{M}^{*}, \rho_{0}, n \in \mathbb{Z}_{+}$. Для того чтобы $S_{n}\left(\beta, \rho_{0}\right)$ было непусто, необходимо и достаточно, чтобы либо $[\beta]$, либо $[\beta]^{-1}$ било представимо в виде $\left[\beta^{+}\right]\left[\beta_{p}\right]\left[\beta_{r}\right][\delta]$, əде $\left[\beta^{+}\right] \in \pi^{+}(A, a),\left[\beta_{p}\right] \in \pi_{p}^{n_{1}}(A, a),\left[\beta_{r}\right] \in \pi_{r}^{n_{2}}(A, a),[\delta] \in\left[\operatorname{im}\left(\psi_{\sharp}\right), \operatorname{im}\left(\psi_{\sharp}\right)\right]$, nричем $\operatorname{deg}[\delta]=\rho \leqslant \rho_{0} \quad u n_{1}+n_{2}=n$.

Доказательство основано на том, что вместо накрытия $p: \bar{M} \rightarrow N$ неориентируемой поверхности можно рассмотреть накрытие $\varphi: \bar{M} \rightarrow \widetilde{N}$ ориентируемой поверхности (см. диаграмму (1)) и воспользоваться результатами из [2], [3].

Итак, исходная задача сведена к чисто алгебраической задаче о факторизации гомотопического класса $[\beta]$ данной кривой $\beta$ в группе $\pi_{1}(A, a)$ (отметим, что эта группа является свободной, что существенно облегчает задачу). Проверка условий теоремы на практике в общем случае представляет собой открытую проблему. Тем не менее, в случае $n=0$ можно предложить алгоритм, позволяющий за конечное число шагов определить, допускает ли $[\beta]$ факторизацию нужного вида. Этот алгоритм основан на модификации понятия базисного набора, введенного в [2], а также на топологическом методе определения степени элемента из коммутанта свободной группы, предложенном независимо Каллером [6] и Ольшанским [7].

\section{СПИСОК ЦИТИРОВАННОЙ ЛИТЕРАТУРЫ}

1. Стоилов С. Лекции о топологических принципах теории аналитических функций. М.: Наука, 1964. 2. Насыров С. Р. // Докл. АН СССР. 1987. Т. 297. №6. С. 1311-1314. 3. Насыров С. Р. // Алгебра и анализ. 1993. Т. 5. № 3. C. 212-236. 4. Ezell C. L. // Trans. Amer. Math. Soc. 1980. V. 259. № 2. P. 533-546. 5. Nasyrov S. R. // Rev. Roumaine Math. Pures Appl. 1995. V. 40. № 2. P. 177-194. 6. Culler M. // Topology. 1981. V. 20. Р. 133-145. 7. Ольшанский А. Ю. // Сиб. матем. ж. 1989. Т. 30. №6. С. 152-171.

Казанский государственный университет 